\title{
Comparative Analysis of the Mechanical Behaviour of PEF and PET Uniaxial Stretching Based on the Time/Temperature Superposition Principle
}

\author{
Emilie Forestier ${ }^{1}\left(\mathbb{D}\right.$, Christelle Combeaud ${ }^{1, *} \mathbb{\infty}$, Nathanael Guigo ${ }^{2}$, Guillaume Corvec ${ }^{1}$, Christophe Pradille ${ }^{3}$, \\ Nicolas Sbirrazzuoli ${ }^{2, *(1)}$ and Noelle Billon ${ }^{1}$ (D)
}

1 MINES Paris Tech, PSL Research University, CNRS, Centre de Mise en Forme des Matériaux (CEMEF), UMR 7635, CEDEX, 06904 Sophia Antipolis, France; emilie.forestier@mines-paristech.fr (E.F.); guillaume.corvec@mines-paristech.fr (G.C.); noelle.billon@mines-paristech.fr (N.B.)

2 Institut de Chimie de Nice (ICN), Université Cote d'Azur, CNRS, UMR7272, CEDEX 2, 06108 Nice, France; nathanael.guigo@univ-cotedazur.fr

3 Mat Xper, 19 Traverse du Barri, 06560 Valbonne, France; christophe.pradille@mat-xper.com

* Correspondence: christelle.combeaud@mines-paristech.fr (C.C.); nicolas.sbirrazzuoli@univ-cotedazur.fr (N.S.)

\section{check for}

updates

Citation: Forestier, E.; Combeaud, C.; Guigo, N.; Corvec, G.; Pradille, C.;

Sbirrazzuoli, N.; Billon, N.

Comparative Analysis of the

Mechanical Behaviour of PEF and PET Uniaxial Stretching Based on the Time/Temperature Superposition Principle. Polymers 2021, 13, 3295. https://doi.org/10.3390/ polym13193295

Academic Editor: Victor Tcherdyntsev

Received: 7 September 2021

Accepted: 23 September 2021

Published: 27 September 2021

Publisher's Note: MDPI stays neutral with regard to jurisdictional claims in published maps and institutional affiliations.

Copyright: (c) 2021 by the authors. Licensee MDPI, Basel, Switzerland. This article is an open access article distributed under the terms and conditions of the Creative Commons Attribution (CC BY) license (https:// creativecommons.org/licenses/by/ $4.0 /)$
Abstract: Poly(ethylene 2,5-furandicarboxylate), PEF and poly(ethylene terephthalate), PET, are two polyesters with close chemical structures. It leads to similar thermal, mechanical and barrier properties. In order to optimize their stretching, a strategy based on the time/temperature principle is used. The building of master curves, in the linear visco-elastic domain, allows the identification of the experimental conditions for which the two materials are in the same physical state. The initial physical state of the materials is important as, to fit with the industrial constrains, the polymers must reach high level of deformation, and develop strain induced crystallization (SIC). In this paper, the screening of the forming range is described, as well as the mechanical response depending on the stretching settings. Moreover, the same mechanical response can exist for PEF and PET if the same gap from the $\alpha$-relaxation exists.

Keywords: biobased thermoplastics; poly(ethylene 2,5-furandicarboxylate) (PEF); poly(ethylene terephthalate) (PET); time/temperature principle; strain-induced crystallization; uniaxial stretching

\section{Introduction}

Poly(ethylene 2,5-furandicarboxylate), PEF and poly(ethylene terephthalate), PET, are two polyesters of rather equivalent mechanical, thermal and barrier properties [1-9]. PEF is considered as the bio-based counterpart of PET that could be used in industrial applications such as stretching, spinning or blowing. Despite these similitudes, they behave slightly differently because of their chain architecture which makes their comparison of high scientific interest. Indeed, the main structural difference between PET and PEF is the presence of the benzene ring in the PET constitutive unit, while it is a furan ring for PEF. It induces different local mobility: contrary to the benzene ring, the activation of the flipping motion of the furan ring is more difficult [1]. Nevertheless, when they are stretched from the amorphous state, both materials can exhibit strain induced crystallization and a drastic mechanical strain hardening [10-12]. It can be achieved when the materials are stretched in a rubbery-like state, where stretch ability is maximum. In industrial processes such as films stretching, injection stretch blow molding (ISBM) or thermoforming, polymers must undergo draw ratio of 4, or more, in two directions (biaxial extensions of 8 to 12). Strain hardening and the ability to develop strain induced crystallization (SIC) are key issues that must be controlled in such processes. Therefore, the ability of these two polyesters for being stretched to high levels, and their ability to develop SIC need a comparison to better understand the general behaviour of aromatic polyesters. 
This phenomenon was widely explored for PET [13-26] since the 70's. There are much less investigations concerning PEF whose technical development is very recent [10-12,27-30]. In PET, SIC is progressively developed $[19,21,22,25,31]$. It is supposed that, before the crystal apparition, there is the existence of an intermediate phase, named mesophase. This oriented and organized phase acts as a crystal precursor [19,21,32-38]. The stable crystal, with all its symmetry and periodicities, needs a relaxation step to appear, and is formed after the stretching end. The mesophase is formed from the stretching beginning [32,34,35,39]. Thus, the obtained microstructure is directly dependent on the stretching settings and on the post-stretching treatment [40]. In PEF, the presence of a mesophase prior to crystallization is postulated [27], but according to our recent works [10-12], we have suggested that PEF microstructural development is more binary: crystal may or may not exist. The crystalline structures are reported to be triclinic for PET [27] and monoclinic for PEF [28,29]. In conditions leading to SIC, the macroscopic behaviour of the polymers is sensitive to the temperature and the strain rate, in a very significant and combined manner (see for example [41]). Additionally, as polymers are not crosslinked, their behaviour results from the combination of hyper-elasticity (as expected in that range) and of viscoelasticity. Consequently, the stretching range has to be defined in terms of temperature/strain rate sets of conditions $(T, \hat{\varepsilon})$. It was already reported that close to $T_{\alpha}$ the combined dependencies of temperature and strain rate could be considered via an unified manner, based on the extension of classical time/temperature superposition principle for different materials (PMMA, PA66, PET and PEF) [10-12,30,40-44]. However, due to differences in the $\alpha$-transition temperature between PEF and PET, the precise processing range must be adjusted to account for the intrinsic chain dynamic. This paper aims at assessing the stretch abilities of amorphous PEF and PET, by extending and validating the "time/temperature" superposition principle identified in the low deformation domain. DMTA analysis allows the building of elastic modulus master curve which could be representative of the polymer behaviour in the large deformation domain. The strategy used is well described in [41]. In a first time, the mechanical behaviour of each material has been investigated by DMTA, where master curves for a reference temperature have been built up. In a second time, cold crystallization in quiescent conditions has been investigated to be certain that, over the stretching duration, the polymer remains totally amorphous. DSC analyses were performed in that way. Then, to assess the stretch abilities of amorphous PET and PEF six different sets of strain rate and temperature were used to perform uniaxial tension up to high deformations and until rupture. Those sets were chosen to address three different equivalent strain rates at a reference temperature, not far from $T_{\alpha}$. The objective is to scan conditions within the entire rubbery-like zone observed on the elastic modulus master curve associated to each polymer. By doubling the technological sets of each equivalent strain rate at the reference temperature, one expects to validate the time/temperature superposition principle at high strains. By using three different equivalent strain rates, corresponding to three different positions on the master curve, one expected to scan most of the processing range that could be found in industrial processes. Digital Image Correlation (DIC) was used to address true tensile stress and strain. The stretching protocol proposed is defined to observe the strain hardening apparition (its onset is defined by the natural draw ratio, NDR), and possibly to allow the materials to develop an organized and oriented microstructure.

Finally, after the stretching, the samples are air-quenched and to complete this study, an overview of the induced microstructures is presented.

\section{Materials and Methods}

\subsection{Materials}

Poly(ethylene 2,5-furandicarboxylate) was synthetized from the direct esterification and melt- solid state polycondensation (SSP) of monoethylene glycol and 2,5-furandicarboxylic acid (FDCA) produced by Avantium Renewable Polymers (Amsterdam, Netherlands). Extruded PEF sheets with a thickness of $700 \mu \mathrm{m}$ have been provided. Samples were extracted in the extrusion direction to minimize thickness variation. A commercially available 
PET grade (RamaPET N180 ${ }^{\circledR}$ from Indorama, Bangkok, Thailand), supplied by Sidel company (Le Havre, France), was extruded into PET sheets by Avantium Renewable Polymers (Amsterdam, the Netherlands) with a thickness of $700 \mu \mathrm{m}$. Samples were extracted in the extrusion direction too.

Extrusions were performed according to state of the art after drying to avoid hydrolysis and degradation. Samples were stored under vacuum, in an aluminium coated bag, in the freezer $\left(-18{ }^{\circ} \mathrm{C}\right)$ to avoid water absorption and physical aging. Consequently, materials were tested dry, as processed, without any pre-conditioning.

\subsection{DMTA Measurements}

All DMTA experiments were conducted in tension using a Mettler-Toledo ${ }^{\circledR}$ DMA 1 , Greifensee, Switzerland. The sample dimensions were $5 \times 4 \times 0.7 \mathrm{~mm}^{3}$ for amorphous samples, and around $5 \times 3 \times 0.3 \mathrm{~mm}^{3}$ for stretched samples. Before each test, the sample underwent a preload of $1 \mathrm{~N}$. Temperature scans were performed between $-150{ }^{\circ} \mathrm{C}$ and $200^{\circ} \mathrm{C}$, at a heating rate of $1{ }^{\circ} \mathrm{C} / \mathrm{min}$, with a displacement amplitude of $5 \mu \mathrm{m}$ (i.e., strain of $0.1 \%$ ), in Auto-Tension mode. Temperature scans were carried out at a frequency of $1 \mathrm{~Hz}$. Three measurements have been performed for each mechanical tests, the curves were well superimposed.

\subsection{DSC Measurements}

DSC measurements are performed on a Mettler Toledo ${ }^{\circledR}$ DSC 1 (Greifensee, Switzerland) equipped with the $\mathrm{STAR}^{\circledR}$ software. Aluminium pans of $40 \mu \mathrm{L}$ are used. The samples weight is of approximatively $3 \mathrm{mg}$.

From their glassy state, the amorphous samples have been submitted to isothermal programs of various temperatures (between $90^{\circ} \mathrm{C}$ and $180^{\circ} \mathrm{C}$ ) and durations (between $1800 \mathrm{~s}$ and $12,000 \mathrm{~s})$. Then, they have been rapidly quenched $\left(50^{\circ} \mathrm{C} / \mathrm{min}\right)$ and heated at $20^{\circ} \mathrm{C} / \mathrm{min}$ from $25^{\circ} \mathrm{C} 250^{\circ} \mathrm{C}$. To measure crystal ratios, the stretched samples have been heated from $25^{\circ} \mathrm{C}$ to $300{ }^{\circ} \mathrm{C}$ at $10^{\circ} \mathrm{C} / \mathrm{min}$. Equation (1) has been used to calculate the crystal ratio obtained after the melting.

$$
\chi=\frac{\Delta H_{m}}{\Delta H_{m}^{0}}
$$

with $\Delta H_{m}$ the melting enthalpy and $\Delta H_{m}{ }^{0}$ the equilibrium melting enthalpy, taken at $140 \mathrm{~J} \times \mathrm{g}^{-1}$ for PEF and PET $[11,30,45]$.

\subsection{Stretching Conditions Determination}

\subsubsection{Determination of the Forming Range}

To perform efficient stretching on PEF and PET, an original protocol has been established, and previously published [10-12]. More details concerning this protocol are given in the present article.

To fit with the industrial protocol, the stretching has to be performed at intermediate temperatures. It means above the $\alpha$-relaxation temperature, to allow chains mobility, but below the static crystallization occurrence, to only enforce strain induced crystallization. As for PET, PEF static crystallization induces a loss of formability and of transparency of the material, which is not in assessment with the industrial requirements. The forming range corresponds to the rubbery plateau of the materials. To illustrate the available forming range, a temperature scan in DMTA, from $25^{\circ} \mathrm{C}$ to $210^{\circ} \mathrm{C}$ at $1 \mathrm{~Hz}$ and $1{ }^{\circ} \mathrm{C} / \mathrm{min}$, is performed for PEF and PET (Figure 1).

A rapid glance to Figure 1 shows that PEF and PET amorphous rubbery plateau, which are the targeted processing ranges, do not overlap. Consequently, stretching the materials with the same set of conditions (temperature, strain rate) will not permit the efficient comparison of the stretch abilities of the two polymers nor to promote simultaneously SIC. In PEF, the low mobility of the chains and the complex interactions induced by the presence of the furan ring led to a higher $\alpha$-relaxation temperature $\left(T_{\alpha}\right)$, compared 
to PET. The $\alpha$-relaxation temperatures (Figure 1 ), taken at the maximum of the Tan $\delta$ peak, are, respectively, of $80^{\circ} \mathrm{C}$ for PET and of $92^{\circ} \mathrm{C}$ for PEF. In parallel, the peak of the cold crystallization is detected for a temperature close to $110{ }^{\circ} \mathrm{C}$ for PET, and to $160{ }^{\circ} \mathrm{C}$ for PEF [10-12].

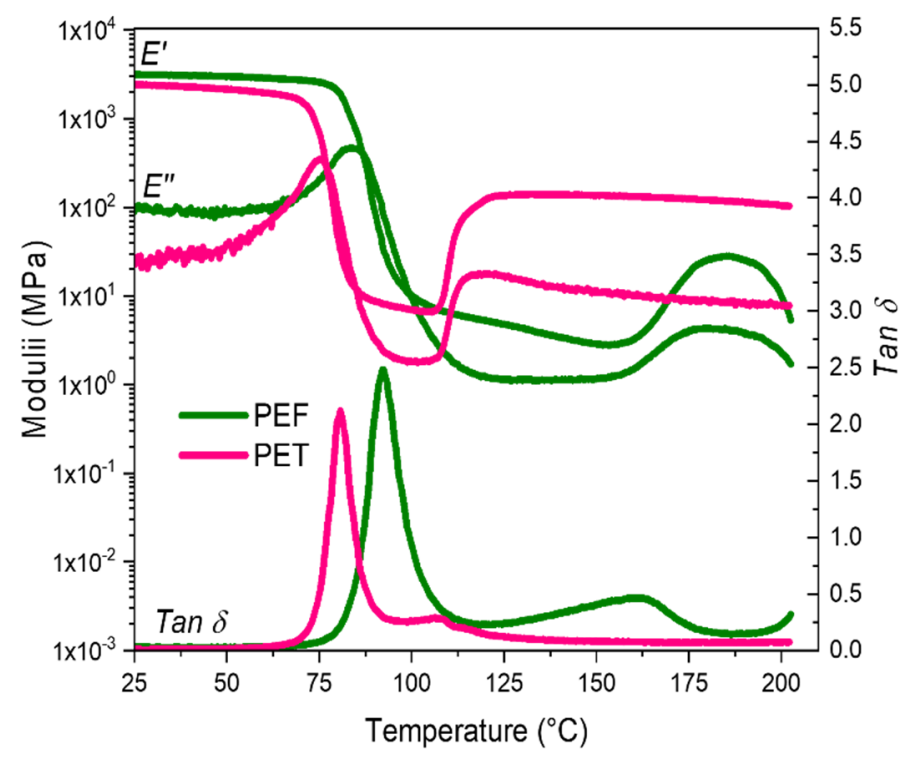

Figure 1. DMTA temperature scans of amorphous PEF (in green) and PET (in pink), for a heating rate of $1^{\circ} \mathrm{C} / \mathrm{min}$ and a frequency of $1 \mathrm{~Hz}$, in a tensile mode [11].

It appears that the cold crystallization in PEF has more difficulty in occurring than the one in PET. It has to be related with the less stability of the former, as demonstrated by the occurrence of the melting right after the static crystallization, at a significant lower melting temperature compared to PET.

Two major differences between PEF and PET must be considered for the stretching:

- $\quad$ PET forming range appears at lower temperature than PEF one;

- PEF exhibits a wider rubbery plateau compared to PET, and consequently a wider forming range.

It means that to perform efficient stretching in PEF, the same settings as those used in PET cannot be relevant.

\subsubsection{Thermal Behaviour in the Forming Range}

Before the stretching, the samples are pre-heated for $5 \mathrm{~min}$. Then, the stretching can be performed at slow or rapid strain rates. It is necessary to be sure that the materials will remain amorphous during the entire test. Thus, an estimate of the time to crystallize as a function of the temperature is needed. It allows to discriminate the sets temperature/maximum duration of test that are relevant or not. To assess heating times, the time needed to induce isothermal crystallization at temperatures included in the forming range has been measured by DSC, and is reported in Figure 2a,b, for, respectively, PEF and PET. To confirm the results of the isotherms, the heating scan of the samples submitted to the isotherms is presented in Figure $3 a, b$, for, respectively, PEF and PET. This heating step allows to estimate whether crystallization has been developed (through the reduction of the cold crystallization enthalpy) or initiated (through the decreasing of the cold crystallization temperature). The measurement error on the crystal ratios is estimated at $\pm 5 \%$. Table 1 reports the evolution of the cold crystallization, melting enthalpies and crystallinity ratios for PEF and PET. As in PEF the isotherms and the following melting of the tests performed at $120^{\circ} \mathrm{C}$ and $130{ }^{\circ} \mathrm{C}$ are spread, these values are not reported. For the same reason, the values of the tests performed at $90^{\circ} \mathrm{C}$ and $95^{\circ} \mathrm{C}$ for PET are not reported. 

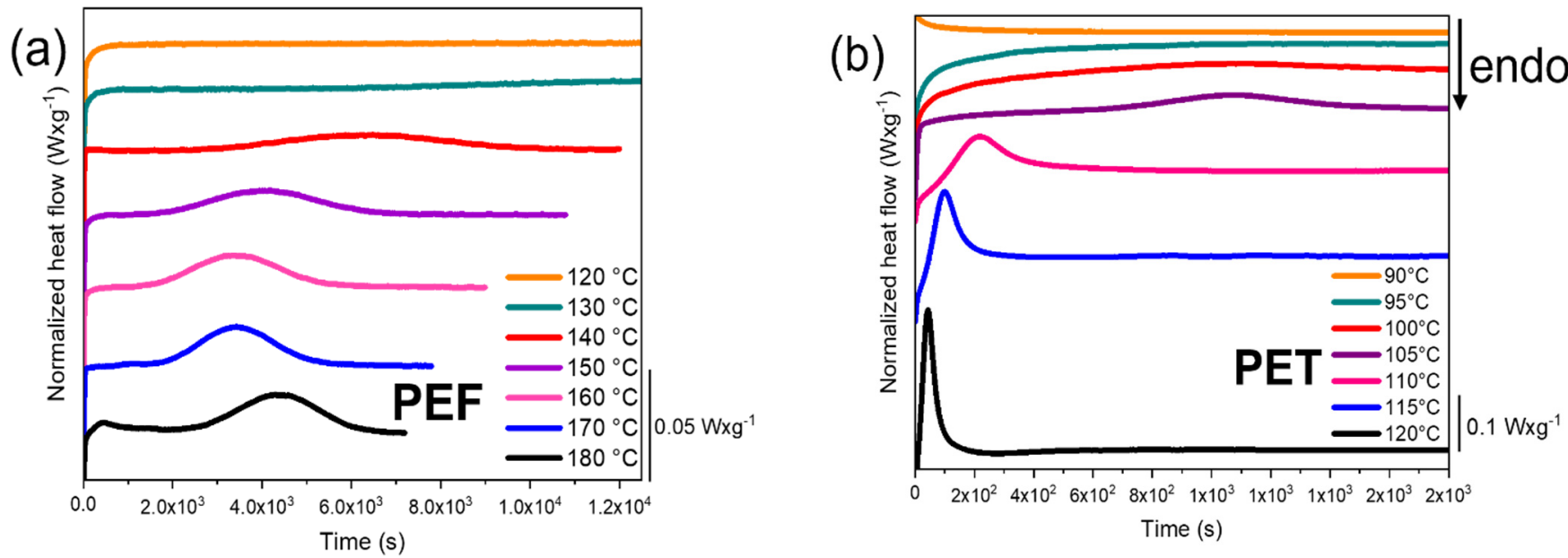

Figure 2. Heating scans, performed at $20^{\circ} \mathrm{C} / \mathrm{min}$, of the previous isothermally crystallized samples for (a) $\mathrm{PEF}$, from $25^{\circ} \mathrm{C}$ to $250^{\circ} \mathrm{C}$ and (b) PET, from $25^{\circ} \mathrm{C}$ from $275^{\circ} \mathrm{C}$, measured by DSC.

(a)

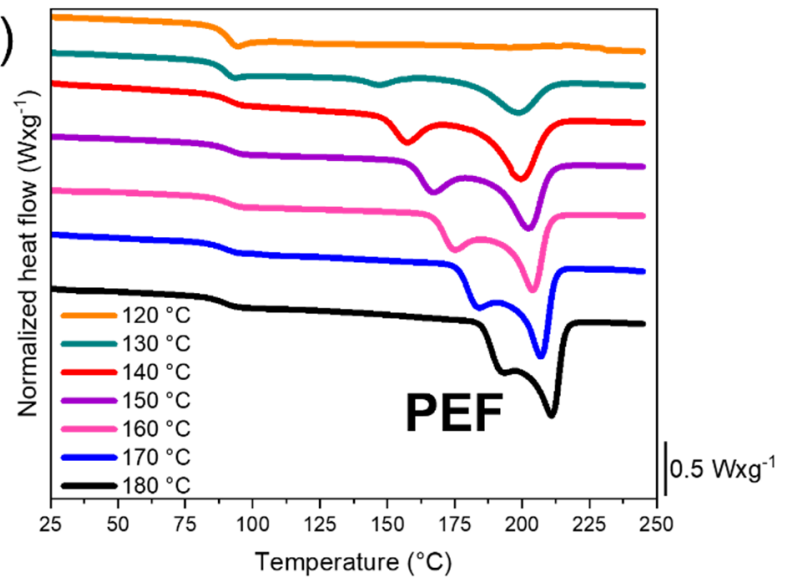

(b)

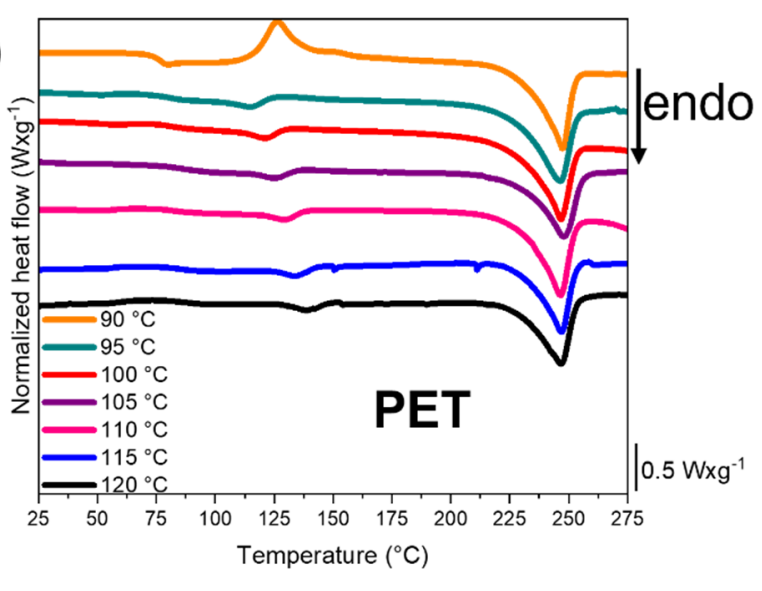

Figure 3. Heating scans, performed at $20^{\circ} \mathrm{C} / \mathrm{min}$, of the previous isothermally crystallized samples for (a) PEF, from $25^{\circ} \mathrm{C}$ to $250{ }^{\circ} \mathrm{C}$ and (b) PET, from $25^{\circ} \mathrm{C}$ from $275^{\circ} \mathrm{C}$, measured by DSC.

Table 1. Evolution of the cold crystallization $\left(\Delta H_{c}\right)$ and melting $\left(\Delta H_{m}\right)$ enthalpies and crystallinity ratios $(\chi)$, during the isothermal treatment and the following melting of PEF and PET.

\begin{tabular}{|c|c|c|c|c|c|c|c|}
\hline \multicolumn{8}{|c|}{ PEF } \\
\hline$T_{\text {isotherm }}\left({ }^{\circ} \mathrm{C}\right)$ & 120 & 130 & 140 & 150 & 160 & 170 & 180 \\
\hline$\Delta H_{c}\left(\mathrm{~J} \cdot \mathrm{g}^{-1}\right)$ & & & 27.69 & 30.24 & 32.38 & 32.09 & 35.57 \\
\hline$\Delta H_{m}\left(\mathrm{~J} \cdot \mathrm{g}^{-1}\right)$ & & & 36.53 & 37.86 & 38.22 & 38.79 & 36.92 \\
\hline$\chi(\%)$ & & & 26.09 & 27.04 & 27.30 & 27.70 & 25.85 \\
\hline \multicolumn{8}{|c|}{ PET } \\
\hline$T_{\text {isotherm }}\left({ }^{\circ} \mathrm{C}\right)$ & 90 & 95 & 100 & 105 & 110 & 115 & 120 \\
\hline$\Delta H_{c}\left(\mathrm{~J} \cdot \mathrm{g}^{-1}\right)$ & & & 7.16 & 12.77 & 9.59 & 9.53 & 12.41 \\
\hline$\Delta H_{m}\left(\mathrm{~J} \cdot \mathrm{g}^{-1}\right)$ & & & 40.13 & 40.58 & 41.08 & 40.65 & 40.53 \\
\hline$\chi(\%)$ & & & 28.66 & 29.00 & 29.34 & 29.03 & 28.95 \\
\hline
\end{tabular}


Concerning PEF, with this experimental protocol, there is no evidence of crystallisation below $130{ }^{\circ} \mathrm{C}$ during the isothermal steps over $12,000 \mathrm{~s}(3 \mathrm{~h})$. At $130^{\circ} \mathrm{C}$, the crystallization peak is spread but visible. For higher temperatures, the static crystallization onset appears to be higher than $1500 \mathrm{~s}$. It is significantly higher than the time needed for the stretching experiments. Looking at the heating traces afterward gives additional information. Up to the isotherm at $120^{\circ} \mathrm{C}$, the heating does not reveal neither cold crystallisation nor fusion. On the contrary, the melting occurs from $130{ }^{\circ} \mathrm{C}$ which means that seemingly some nucleation can develop during the isotherms. The results are in agreement with the investigation of Martino et al. that demonstrated the formation of PEF crystals when exceeding annealing time of $1500 \mathrm{~s}$ and $3000 \mathrm{~s}$ at, respectively, $130{ }^{\circ} \mathrm{C}$ and $120{ }^{\circ} \mathrm{C}$ [46]. In conclusion, the static crystallisation is negligible in PEF up to $120^{\circ} \mathrm{C}$, whatever the test durations are. From that limit, the crystallization could be firstly initiated during the heating step, and then developed during the tensile test.

PET behaves in a different way.

For the measurement realised at $105^{\circ} \mathrm{C}$, it is visible that the isothermal crystallisation is significant from $800 \mathrm{~s}$. At higher temperatures, the isothermal crystallisation occurs faster. PET is still amorphous after $1800 \mathrm{~s}$ at $90^{\circ} \mathrm{C}$, but semi crystalline after $1800 \mathrm{~s}$ at $95^{\circ} \mathrm{C}$ (Figure $3 b$ ). As it was already pointed out in a previous study [47], the static crystallisation of amorphous PET can be neglected up to $90^{\circ} \mathrm{C}$. From $90^{\circ} \mathrm{C}$ to $105^{\circ} \mathrm{C}$, one has to be very rigorous in terms of duration of tests as crystallisation can develop during the tests.

To be complete, the crystal ratios developed after the isotherms and the following meltings are of around $27 \%$ for PEF, and $28 \%$ for PET. It enlightens the fact that the two materials are only different in terms of kinetic of crystallization. Lastly, the melting behaviours are different between PEF and PET. After the isothermal crystallization, PET exhibits one unique melting temperature, while PEF melting appears to be multiple and sensitive to the crystallisation conditions. This trend has already been reported $[5,6,45,48,49]$. The stretching ranges, in terms of temperatures, are known for both materials. The strain rates must, by now, be adjusted and must fit with these temperatures.

\subsubsection{Dependence to the Frequency in the Forming Range}

To define the stretching settings composed of couple strain rate/temperature, differences in $\alpha$-relaxation temperature have to be accounted for. As the aim of this work is to compare the two polymers in an identical physical state, it has been decided to stretch the polymers with different temperatures and strain rates, but at similar equivalent strain rates at a reference temperature chosen close to the respective $\alpha$-transition temperature of the materials. The WLF approach, which is the most common formal approach for time temperature principle, is used. As a result, master curves at a reference temperature are built up for PEF and PET. This approach has already been reported relevant for PEF [10-12,30], PET $[40,50]$ or other materials [41-44].

From a physical aspect, this principle stipulates that the behaviour of the polymer depends in an equivalent manner on the temperature and on the strain rate. It means that starting from a given temperature, $T$, at a given strain rate, $\dot{\varepsilon}$ (or frequency, $f$ ), the mechanical behaviour will change in equivalent manners whether $T$ is increased or $\varepsilon$ is decreased. In a range of temperature close to $T_{\alpha}$, it is often validated that elastic modulus, $E$, is such as demonstrated in Equation (2).

$$
E(T, e)=E\left(T_{r e f}, \dot{\varepsilon} \times a_{T / T_{r e f}}\right)
$$

The so-called shift factor, $a_{T / T r e f}$, only depends on $T$ and $T_{\text {ref }}$, which is the so-called reference temperature. This latter can be arbitrary chosen. WLF formalism for shift factor is reminded in Equation (3) [51].

$$
\log \left(a_{T / \text { Tref }}\right)=\frac{-C_{1}^{0}\left(\mathrm{~T}-T_{r e f}\right)}{C_{2}^{0}+\left(T-T_{r e f}\right)}
$$


with $a_{T / \text { Tref }}$ the shift factor, $C_{1}^{0}$ and $C_{2}^{0}\left({ }^{\circ} \mathrm{C}\right)$ the viscoelastic coefficients, $T$ the temperature and $T_{\text {ref }}$ the reference temperature (in this work $100{ }^{\circ} \mathrm{C}$ and $90{ }^{\circ} \mathrm{C}$ for, respectively, PEF and PET).

The master curves are deduced from isothermal frequency scans, from $1 \mathrm{~Hz}$ to $100 \mathrm{~Hz}$. The temperature step is of $5{ }^{\circ} \mathrm{C}$, from $85^{\circ} \mathrm{C}$ to $135^{\circ} \mathrm{C}$. Only a horizontal shift is applied. These frequency scans, performed in the forming range, are visible for PEF in Figure 4.

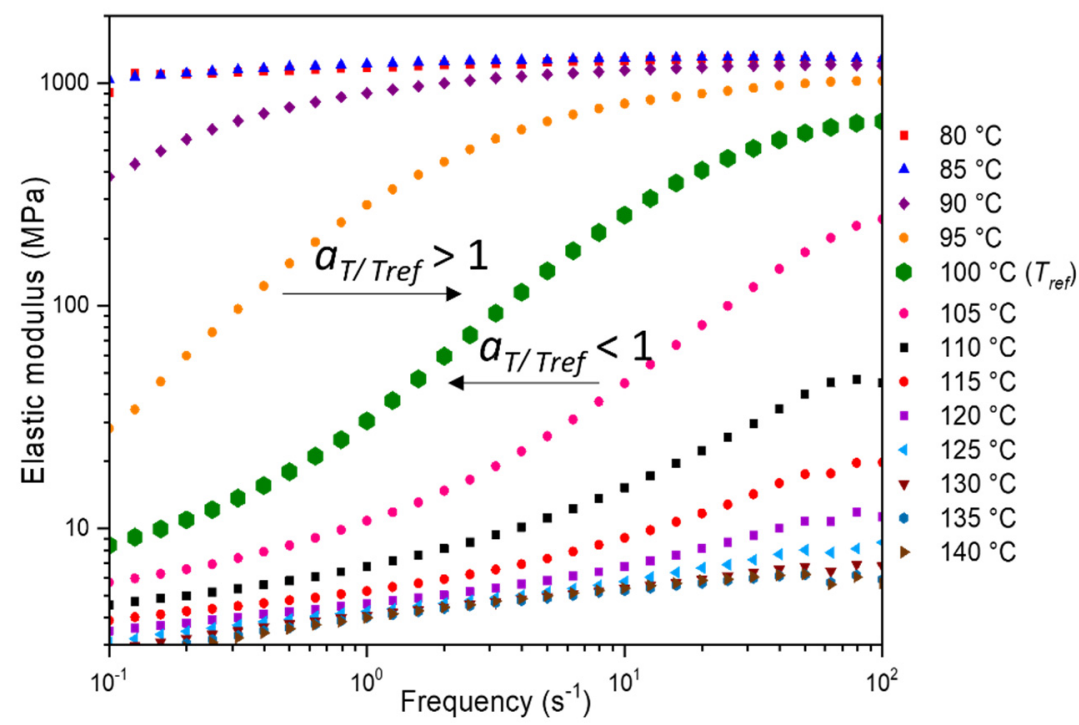

Figure 4. PEF frequency scans in the forming range from $10^{-1} \mathrm{~s}^{-1}$ to $10^{2} \mathrm{~s}^{-1}, a_{T / \text { Tref }}$ is the shift factor.

According to the protocol explained, the typical PEF and PET master curves are given in Figure $5 a, b$ depicts the evolution of the shift factor for both materials depending on the gap from the reference temperature. Figure $5 c, d$ depict the linear regression that validates the WLF formalism of the PEF and PET master curves. From the equation of the linear regression the WLF factors $\left(C_{1}^{0}\right.$ and $\left.C_{2}^{0}\right)$ are obtained. Table 2 summarizes the values of the WLF parameters found.
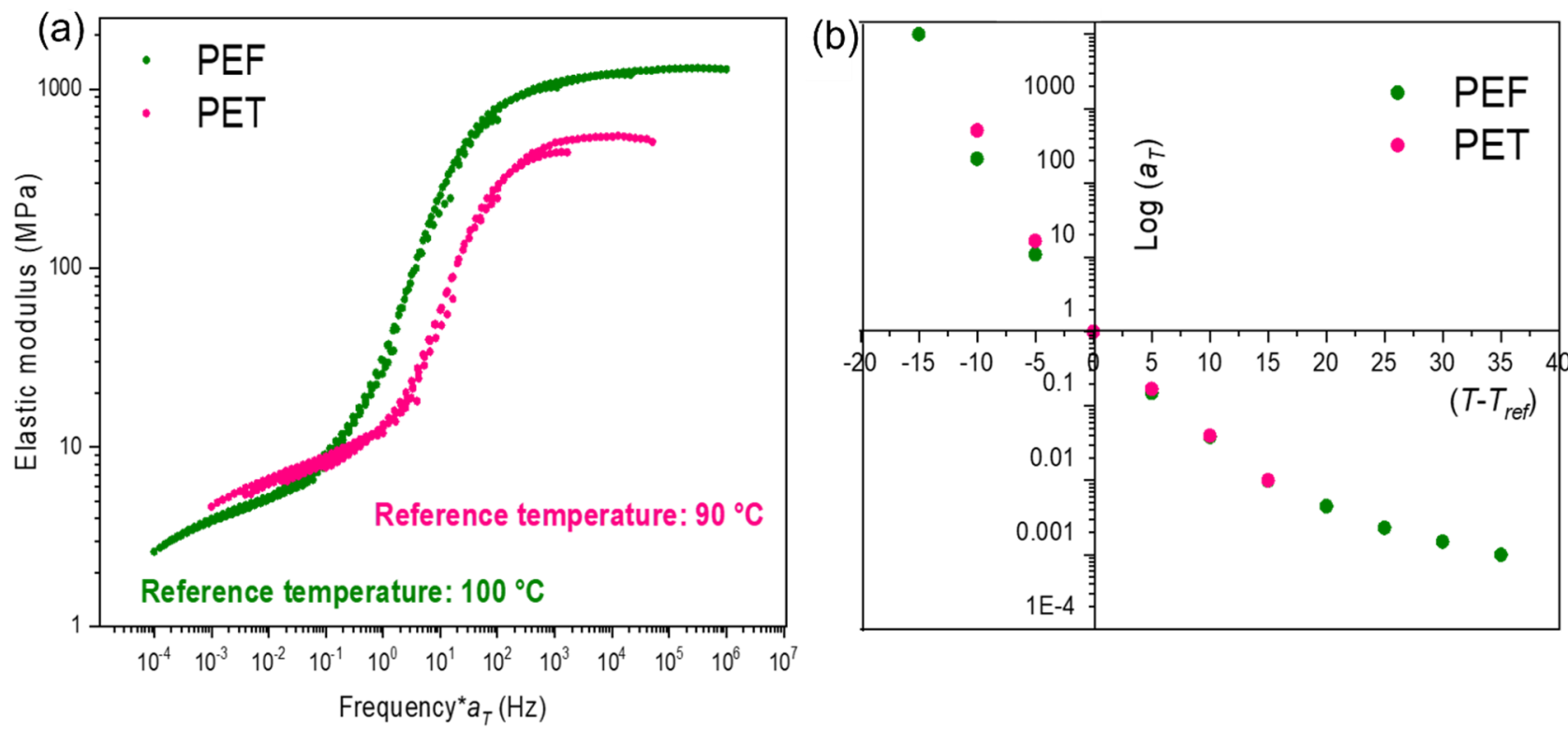

Figure 5. Cont. 

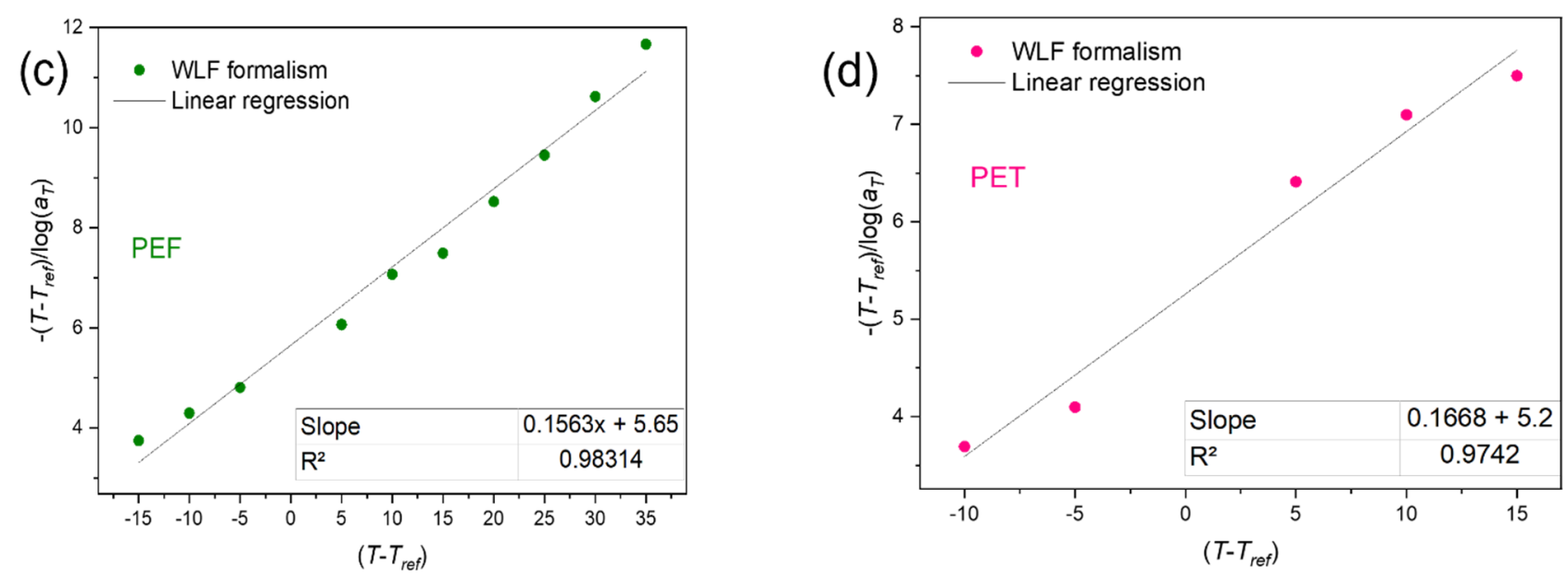

Figure 5. (a) Master curves of PEF and PET at reference temperatures of, respectively, $100{ }^{\circ} \mathrm{C}$ and $90{ }^{\circ} \mathrm{C}$, (b) evolution of the shift factor as a function of the gap from the reference temperature, linear regressions of (c) PEF and (d) PET master curves used for uniaxial stretching.

Table 2. Summary of the reference temperatures, and the viscoelastic parameters of the WLF formalism for PEF and PET.

\begin{tabular}{cccc}
\hline Materials & $\boldsymbol{T}_{\text {ref }}\left({ }^{\circ} \mathbf{C}\right)$ & $C_{1}^{0}$ & $C_{2}^{0}\left({ }^{\circ} \mathbf{C}\right)$ \\
\hline PEF & 100 & 6.39 & 36.18 \\
\hline PET & 90 & 5.99 & 31.52 \\
\hline
\end{tabular}

As previously settled [10-12], PEF and PET master curves are close. It is particularly true for their rubbery plateaux. One can observe the closeness of the $a_{T}$ values, between PEF and PET, once they are expressed as a function of $T-$ Tref (Figure $5 b$ ). The closeness of WLF's parameters is also visible in Table 2. It encourages thinking that the materials could be tested in the same physical state.

\subsection{Mechanical Tests}

\subsubsection{Exploration of the Forming Range}

Once the master curves have been built, the stretching parameters can be chosen. The first step is to select the localization targeted on the master curve, represented by the equivalent strain rate at the reference temperature $\left(\hat{\varepsilon} \times a_{T / \text { Tref }}\right)$. It means the physical state of the material and thus, the gap from the $\alpha$-relaxation. By this way, as this work aims at exploring widely the forming range of PEF and PET, three localizations have been selected on the master curve: beginning of the rubbery plateau (close to the $\alpha$-relaxation), middle and end of the rubbery plateau. These zones are represented in Figure $6 \mathrm{a}, \mathrm{b}$ for, respectively, PEF and PET. As a first approach, in a similar way as the Cox Merz rule [52], it is postulated that if the complex modulus evolves as a function of the frequency, the constitutive parameters of the material should evolve as a function of the strain rate [41].

For each equivalent strain rate, two different technological couples (strain rate/temperature) have been chosen. One condition is named "slow" which has to do with the slowest strain rate used, while the other condition is named "rapid" for the fastest strain rate. The aim is to have around one decade of difference on the strain rate between these two tests performed at the same equivalent strain rate. Hence, the time/temperature superposition principle is going to be tested in the large deformation domain.

The stretching settings applied during the tensile tests are, for each equivalent strain rate, summarized in Table 3. 

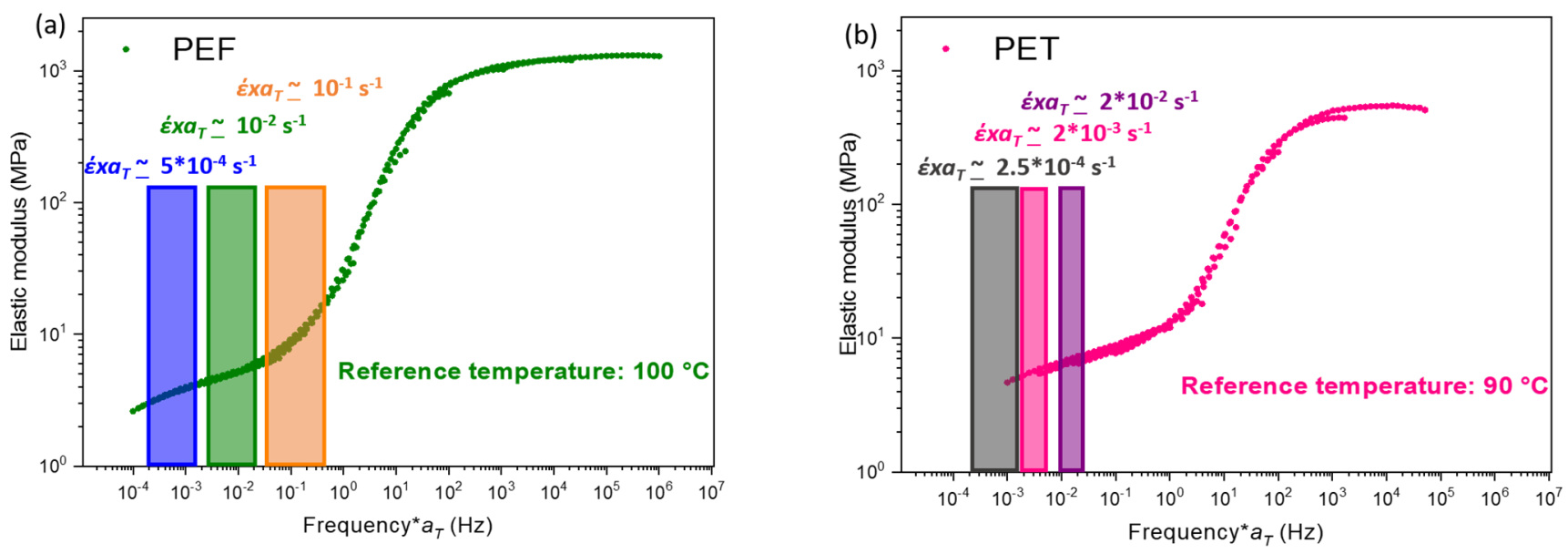

Figure 6. Master curves of (a) PEF and (b) PET with the areas associated to each equivalent strain rate at the reference temperature.

Table 3. Summary of the stretching settings associated to each equivalent strain rate at the reference temperature, for PEF and PET.

\begin{tabular}{|c|c|c|c|c|}
\hline \multirow[t]{3}{*}{ Equivalent Strain Rate (s ${ }^{-1}$ ) } & \multicolumn{2}{|c|}{ Stretching Settings } & \multicolumn{2}{|c|}{ Stretching Settings } \\
\hline & \multicolumn{2}{|c|}{ PEF } & \multicolumn{2}{|c|}{ PET } \\
\hline & $\begin{array}{c}\text { Slow } \\
\left(s^{-1 /}{ }^{\circ} \mathrm{C}\right)\end{array}$ & $\begin{array}{c}\text { Rapid } \\
\left(s^{-1 /}{ }^{\circ} \mathrm{C}\right)\end{array}$ & $\begin{array}{c}\text { Rapid } \\
\left(s^{-1 /}{ }^{\circ} \mathrm{C}\right)\end{array}$ & $\begin{array}{c}\text { Rapid } \\
\left(s^{-1 /}{ }^{\circ} \mathrm{C}\right)\end{array}$ \\
\hline $10^{-1}$ & $0.02 / 96$ & $0.20 / 101$ & & \\
\hline $2 \times 10^{-2}$ & & & $0.01 / 87$ & $0.06 / 92$ \\
\hline $10^{-2}$ & $0.035 / 101$ & $0.130 / 109$ & & \\
\hline $2 \times 10^{-3}$ & & & $0.02 / 95$ & $0.07 / 101$ \\
\hline $5 \times 10^{-4}$ & $0.015 / 109$ & $0.040 / 117$ & & \\
\hline $2.5 \times 10^{-4}$ & & & $0.009 / 101$ & $0.030 / 106$ \\
\hline
\end{tabular}

\subsubsection{Stretching Device and Sample Geometry}

As explained in previous works [10-12], a homemade device designed for film stretching under controlled temperature conditions was used. It can reproduce industrial uniaxial and biaxial stretching conditions. It is composed of four independent motor-driven arms, each coupled to a displacement sensor and a $500 \mathrm{~N}$ force transducer. Tensile velocity was ruled to keep strain rate, $\dot{\varepsilon} 0$, as constant as possible in the central zone of the samples. To this purpose, velocity of the arms varies exponentially with time.

The sample can be heated and, after being stretched, annealed or quenched with several mobile ovens. A window of zinc selenide ( $\mathrm{ZnSe})$, which is partially transparent to infrared radiations, allows to measure the specimen surface temperature during the tests. On the other side of the sample, another borosilicate glass window allows local measurements of displacement fields using DIC (2D Digital Image Correlation) on painted speckle. Strain fields are computed from the displacement fields. It was shown that adding a painted speckle of thickness of almost $40 \mu \mathrm{m}$ did not impact force measurement. DIC was used to address local Hencky's strain $\varepsilon_{x x}$ and $\varepsilon_{y y}$, on the specimen surface and in the two directions, longitudinal and transversal, respectively. All measurements were performed at the same location on the sample, i.e., the central zone where local stress and strain were measured. Figure 7a shows this localization, as well as an example of strain field obtained with DIC2D. Figure $7 \mathrm{~b}$ represents the geometry of the samples. 

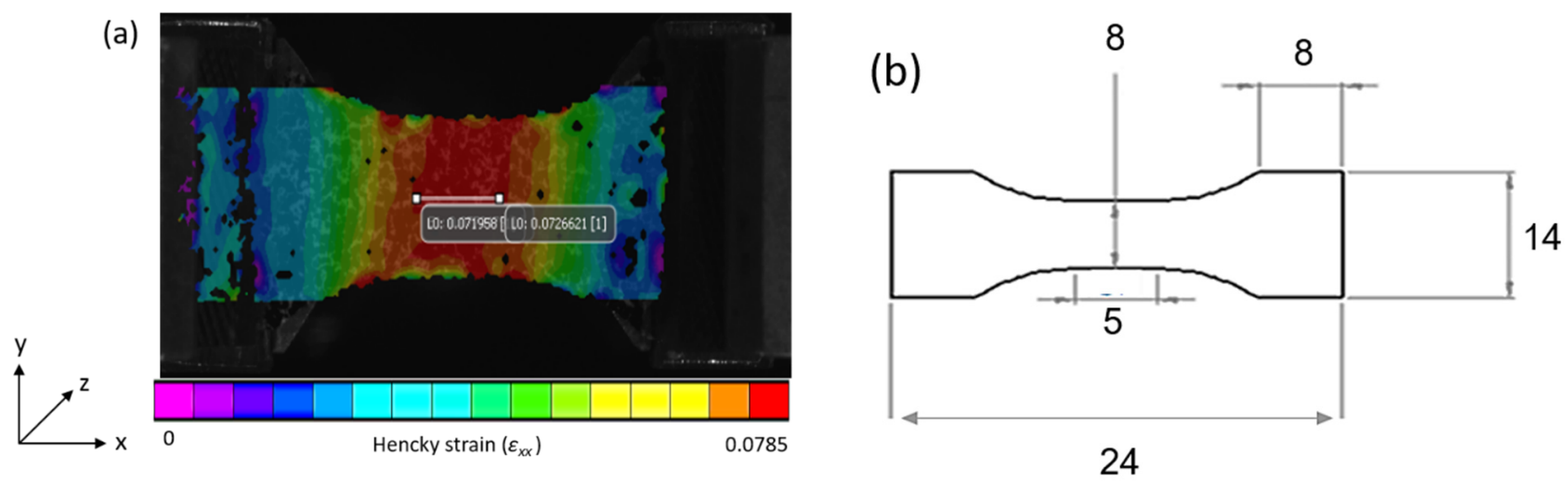

Figure 7. (a) Measurement of Hencky strain field, $\varepsilon_{x x}$, during a tensile test performed at $101{ }^{\circ} \mathrm{C}$ and $0.035 \mathrm{~s}^{-1}$ for PEF, (b) Dimensions of the sample (in $\mathrm{mm}$ ).

Mechanical tests were analysed in terms of true stress (calculated using the actual instantaneous section) and true strain as depicted by Equation (4). Transverse isotropy hypothesis was assumed $[10,11]$.

$$
\sigma(t)=\frac{F(t)}{e_{0} \times w_{0} \times \exp \left(2 \varepsilon_{y y}(t)\right)}
$$

with, $w_{0}$ and $e_{0}$ the initial width and thickness, and $F(t)$ the force evolution with time.

For each measurement, an IR pyrometer and a CCD camera were synchronised to the other analogic signals (force, displacement ... ). The paint was mechanically removed for post-stretching analysis. In any case, material was quenched after drawing to freeze the microstructure.

\section{Results}

\subsection{Mechanical Behaviour}

Figure 8a represents the mechanical responses obtained for both PEF and PET. For a better reading of the initial steps of the stretching, a zoom is shown in Figure $8 \mathrm{~b}$. For the same reason, curves are redrawn in Figure 9, material by material.
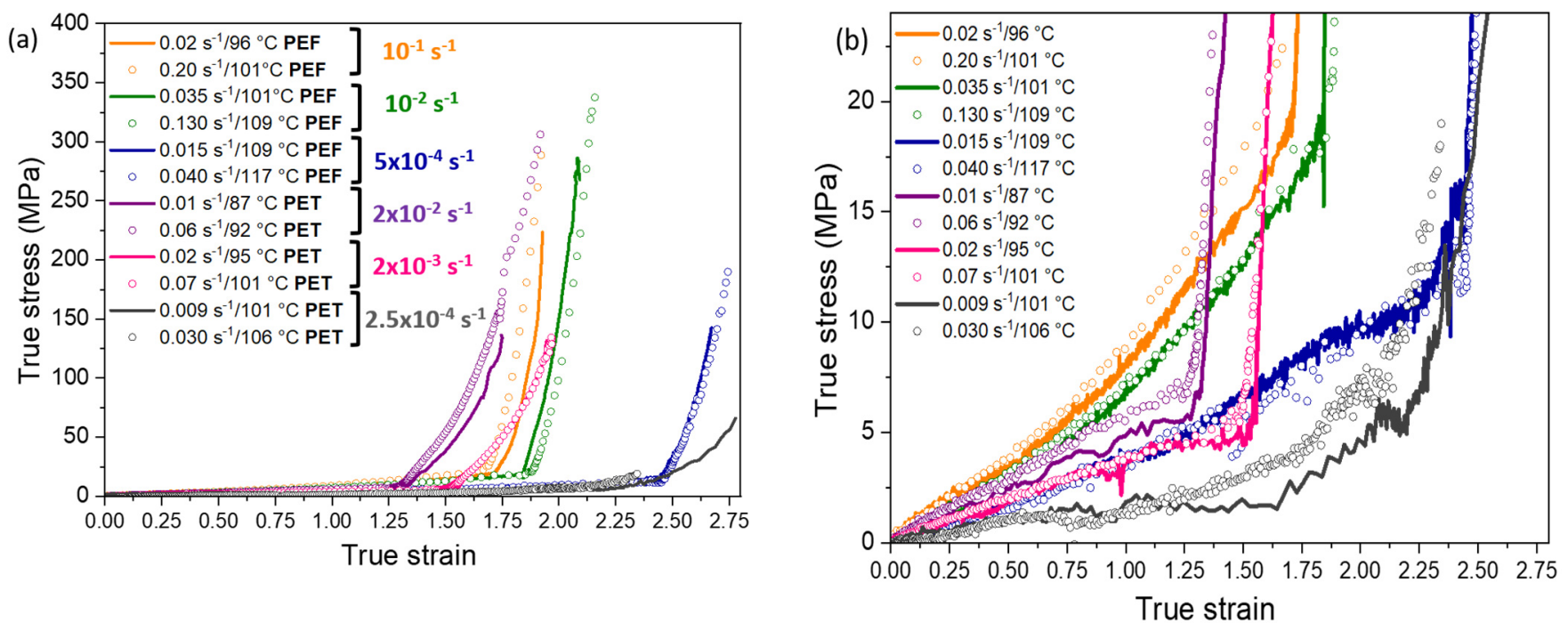

Figure 8. (a) True stress/strain curves of uniaxially stretched PEF and PET. Each colour is associated to an equivalent strain rate. Within an equivalent strain rate, lines are for "slow" tests while dots belong to "rapid" tests; (b) a zoom is made on the first deformation stages. 

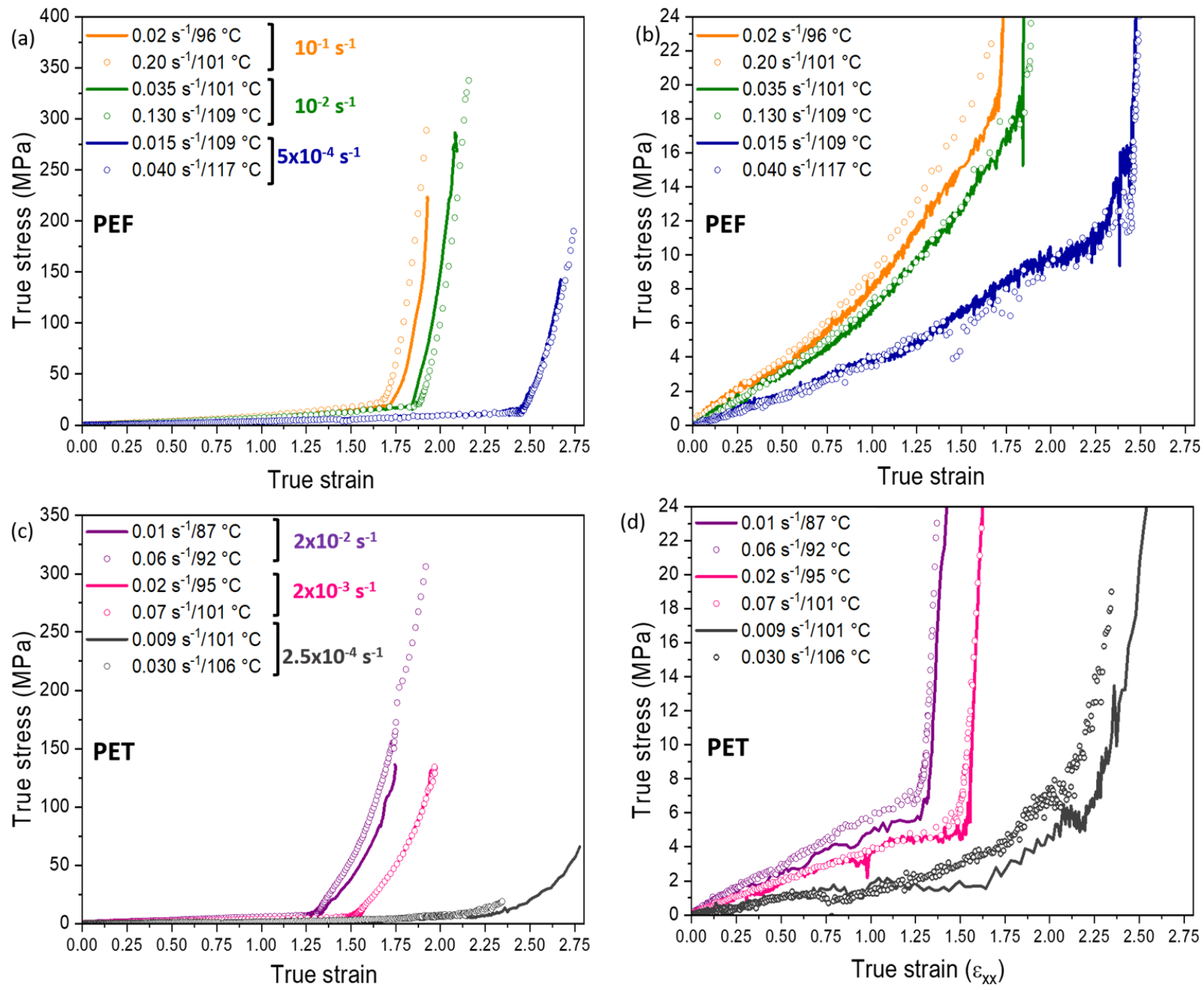

Figure 9. True stress/strain curves for (a) and (b) PEF, (c) and (d) PET.

The mechanical response is governed by the equivalent strain rate. The lower the equivalent strain rate, the lower the slope at the curve origin. The first stages of the stretching belong to the elastic domain. Then, the slope at the curve origin represents the Young modulus that decreases when the rubbery state of the material increases.

The stretching protocol leads to six mechanical tests for each material. Each mechanical response exhibits an impressive strain hardening up to the rupture (Figure 8). The progressive increase of the stress is due to the extension of the chains. For each equivalent strain rate tested, the stress-strain curves associated to two different couples (strain rate/temperature) are superimposed. The time/temperature principle is validated for all the conditions. It allows a potential transposition of the results to the industry that uses faster strain rates, and then higher temperatures. For the PET samples stretched with an equivalent strain rate equal to $2.5 \times 10^{-4} \mathrm{~s}^{-1}$ (grey curves), some differences exist concerning the strain hardening onset between the two tests. For the stretching performed at $106^{\circ} \mathrm{C}$ (highest temperature), it is possible that some nucleation has occurred during the pre-heating step and the test itself. In total, this sample has been heated above its $\alpha$-relaxation during $335 \mathrm{~s}$. Nevertheless, the isothermal tests performed in DSC close to this temperature (shown in Section 2.3) have reported no crystallization. However, the strain hardening development is complex and singular in comparison with the other tests. According to the mechanical behaviour of this sample (early apparition of the NDR, and low strain hardening level), the possible nucleation during the pre-heating step should be considered. 
As shown in Figure 8b, the behaviour of PEF and PET appears really close during the first steps of the tests (except for PET stretched with an equivalent strain rate of $2.5 \times 10^{-4} \mathrm{~s}^{-1}$ ). Up to around a strain of 1.3, PEF test performed at the beginning of the rubbery plateau $\left(10^{-1} \mathrm{~s}^{-1}\right.$, orange curves) exhibits the stiffer behaviour. The less rigid tests are those of PET which are localized right before the static crystallization $\left(2.5 \times 10^{-4} \mathrm{~s}^{-1}\right.$, grey curves). Between them, the other tests are close.

A superimposition is even noticeable between PEF stretched at an equivalent strain rate of $5 \times 10^{-4} \mathrm{~s}^{-1}$ (blue curves), and PET stretched at an equivalent strain rate of $2 \times 10^{-3} \mathrm{~s}^{-1}$ (pink curves). These observations confirm that it is the gap from the $\alpha$-relaxation that determines the mechanical behaviour. Indeed, to acquire the same localization on the PEF and PET rubbery plateau, and to obtain a similar response during the first stages of the stretching, around one decade of difference has to be applied on the equivalent strain rates defined at the reference temperature close to $T_{\alpha}$. It explains the superimposition of the tests performed at equivalent strain rates of, respectively, $5 \times 10^{-4} \mathrm{~s}^{-1}$ and $2 \times 10^{-3} \mathrm{~s}^{-1}$ for, respectively, PEF and PET.

Table 4 gathers the NDRs of each experiment, as well as the Hencky's strain at the NDR, for PEF and PET. As the NDR apparition is relatively abrupted, the value has been determined directly at the break in slope. The reaching of high draw ratios is definitely visible.

Table 4. NDR evolution with the equivalent strain rates at the reference temperature, for PEF and PET.

\begin{tabular}{|c|c|c|c|c|c|c|}
\hline \multicolumn{7}{|c|}{ PEF } \\
\hline$\varepsilon \times a_{T}$ & \multicolumn{2}{|c|}{$10^{-1} \times s^{-1}$} & \multicolumn{2}{|c|}{$10^{-2} \times s^{-1}$} & \multicolumn{2}{|c|}{$5 \times 10^{-4} \times s^{-1}$} \\
\hline $\begin{array}{l}\text { Settings } \\
\left(\mathrm{s}^{-1} /{ }^{\circ} \mathrm{C}\right)\end{array}$ & $0.02 / 96$ & $0.20 / 101$ & $0.035 / 101$ & $0.130 / 109$ & $0.015 / 109$ & $0.040 / 117$ \\
\hline Hencky's strain at NDR & 1.69 & 1.68 & 1.83 & 1.87 & 2.39 & 2.40 \\
\hline $\operatorname{NDR}(\lambda)$ & 5.41 & 5.36 & 6.29 & 6.48 & 11.02 & 11.13 \\
\hline \multicolumn{7}{|c|}{ PET } \\
\hline$\dot{\varepsilon} \times a_{T}$ & \multicolumn{2}{|c|}{$2 \times 10^{-2} \times s^{-1}$} & \multicolumn{2}{|c|}{$2 \times 10^{-3} \times s^{-1}$} & \multicolumn{2}{|c|}{$2.5 \times 10^{-4} \times \mathrm{s}^{-1}$} \\
\hline $\begin{array}{l}\text { Settings } \\
\left(\mathrm{s}^{-1} /{ }^{\circ} \mathrm{C}\right)\end{array}$ & $0.01 / 87$ & $0.06 / 92$ & $0.02 / 95$ & $0.07 / 101$ & $0.009 / 101$ & $0.030 / 106$ \\
\hline Hencky's strain at NDR & 1.27 & 1.25 & 1.53 & 1.47 & 2.19 & 2.05 \\
\hline $\operatorname{NDR}(\lambda)$ & 3.56 & 3.49 & 4.61 & 4.34 & 8.93 & 7.76 \\
\hline
\end{tabular}

The NDR apparition is dependent on the equivalent strain rate (and on the chain mobility). Its occurrence takes place at higher strains when the rubbery state of the material is more marked. Moreover, the NDR appears always at higher strains for PEF compared to PET, even for close localizations on their rubbery plateau. Furthermore, the strain hardening development seems sharper in PEF compared to PET. According to one of our previous works [11], the need of PEF to reach higher NDR can be due to its need to form firstly a crystal before the strain hardening occurrence. Thus, PEF is stretched and the chains have a low mobility, no intermediary phases can exist and the crystal appears only when the conditions of appearance are satisfied: (i) change of the ethylene glycols from gauche to trans (ii) the conformation of furans from anti to syn, (iii) need of two repeating units in its crystal $[12,29,53])$

The results obtained are in good agreement with the previous works concerning PET $[11,21,40,50]$. It confirms the interest of using a master curve to estimate the physical state of the material before its stretching, and then to apply the adequate couple strain rate/temperature. With the few tests chosen from the master curve, the mechanical behaviour of PEF and PET can be widely described.

The definition of the PEF stretching settings from the master curve analysis is more efficient than the choices existing in the literature [27-29]. In these previous works, PEF was 
not able to reach high level of deformations, nor to develop high level of strain hardening (the stress levels were lower than $10 \mathrm{MPa}$ ), and then, the induced microstructure was not a well-defined one. It is probably due to the use of a too low temperature and strain rate.

To conclude this part, the use of the protocol described in the present paper leads to control tests. It is observed that PEF and PET stretched with the relevant settings are not so different in terms of mechanical behaviours.

\subsection{Induced Microstructure}

The creation of SIC is observable on the Debye-Scherrer pictures for PEF and PET (respectively, Figures 10 and 11). Initially, the materials are amorphous and after the stretching, the observation of intense spots is obvious on the patterns. The spots represent the diffraction of the families of planes. It reveals the periodicity of the structure, oriented in the material, and thus the presence of SIC [10-12].

\section{PEF}

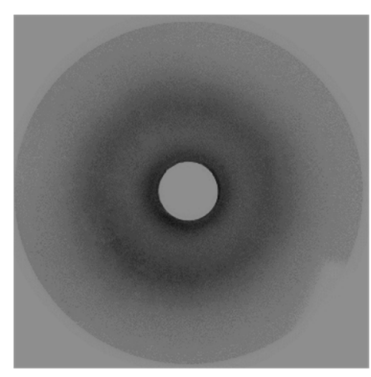

Unstretched PEF
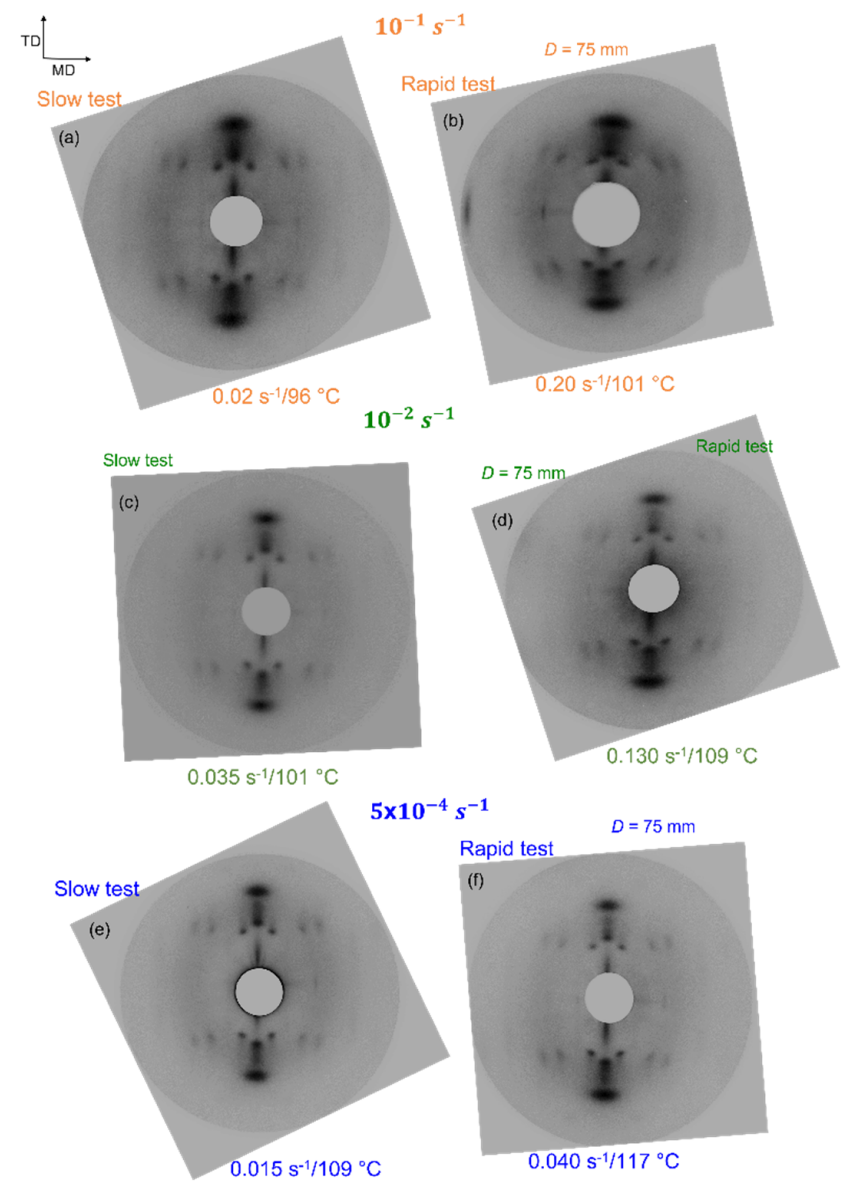

Figure 10. Debye-Scherrer patterns of stretched PEF, with $(\mathbf{a}, \mathbf{c}, \mathbf{e})$ "slow" and $(\mathbf{b}, \mathbf{d}, \mathbf{f})$ "rapid" strain rates. 


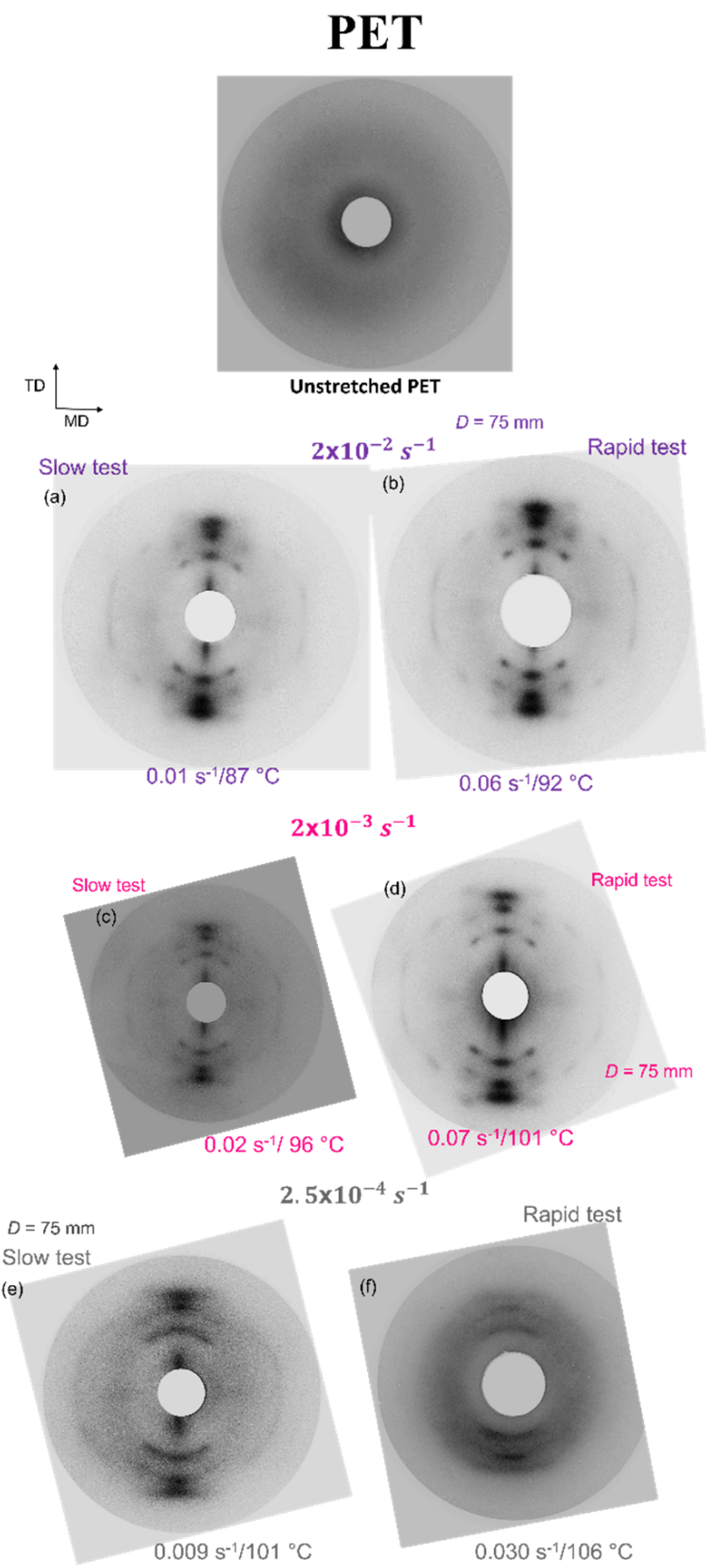

Figure 11. Debye-Scherrer patterns of stretched PET, with $(\mathbf{a}, \mathbf{c}, \mathbf{e})$ "slow" and (b,d,f) "rapid" strain rates.

A well-defined crystal exists for all the stretching conditions. It is especially true for PEF and it validates the stretching protocol efficiency as well as the control of the stretching settings. For PET, two samples (Figure 11e,f) exhibit different patterns in comparison with the other conditions presented. The spots appear spreader and rings are partially visible. The sample stretched at $106^{\circ} \mathrm{C}$ confirms this as arcs rather than spots are visible. Thus, the crystal perfection of these samples can be lower compared to the other tests. PET sample that has been stretched with the highest temperature $\left(106^{\circ} \mathrm{C}\right)$ can have developed a microstructure which is a mix between SIC and some nucleation occurring during the pre-heating step. It is confirmed by the low strain hardening visible in Figure 8, the microstructure of this sample is rather an organized mesophase than a crystal. 


\section{Conclusions}

The aim of this work was to stretch PEF and PET all along their forming range. An original protocol was used to stretch both materials while considering their own chain mobility and thus the gap existing from the $\alpha$-relaxation. The gap from the $\alpha$-relaxation, represented through the equivalent strain rate, is the key parameter that controls the mechanical response and the NDR value.

PEF and PET can be stretched efficiently along their forming range and an important strain hardening has been noticed. SIC has been developed for all the samples. The time/temperature principle has been validated for all the stretching conditions which then allows the transposition of these results in industrial conditions. Moreover, during the first steps of the stretching, all the curves are close, and almost superimposed. It attests of the high similarity existing between PEF and PET. To acquire the same mechanical response, around one decade of difference must exist when the stretching settings are extracted from the master curve reading.

Author Contributions: Data curation, E.F. and C.C.; formal analysis, E.F., C.C., N.G., G.C., C.P., N.S. and N.B.; funding acquisition, C.C., N.G., N.S. and N.B.; methodology, C.C., N.G., N.S. and N.B.; project administration, C.C., N.G., N.S. and N.B.; supervision, C.C., N.G., N.S. and N.B.; validation, E.F., C.C., N.G., N.S. and N.B.; writing—original draft, E.F.; writing—review and editing, C.C., N.G., G.C., C.P., N.S. and N.B. All authors have read and agreed to the published version of the manuscript.

Funding: This work was supported by the French Environment and Energy Management Agency (ADEME) and Sidel as well as Avantium Renewable Polymers.

Institutional Review Board Statement: Not applicable.

Informed Consent Statement: Not applicable.

Acknowledgments: Authors thank the French Environment and Energy Management Agency (ADEME) and Sidel as well as Avantium Renewable Polymers.

Conflicts of Interest: The authors declare no conflict of interest.

\section{References}

1. $\quad$ Burgess, S.K.; Leisen, J.E.; Kraftschik, B.E.; Mubarak, C.R.; Kriegel, R.M.; Koros, W.J. Chain Mobility, Thermal, and Mechanical Properties of Poly(Ethylene Furanoate) Compared to Poly(Ethylene Terephthalate). Macromolecules 2014, 47, 1383-1391. [CrossRef]

2. $\quad$ Burgess, S.K.; Kriegel, R.M.; Koros, W.J. Carbon Dioxide Sorption and Transport in Amorphous Poly(Ethylene Furanoate). Macromolecules 2015, 48, 2184-2193. [CrossRef]

3. Burgess, S.K.; Karvan, O.; Johnson, J.R.; Kriegel, R.M.; Koros, W.J. Oxygen Sorption and Transport in Amorphous Poly(Ethylene Furanoate). Polymer 2014, 55, 4748-4756. [CrossRef]

4. $\quad$ Burgess, S.K.; Mikkilineni, D.S.; Yu, D.B.; Kim, D.J.; Mubarak, C.R.; Kriegel, R.M.; Koros, W.J. Water sorption in Poly(Ethylene Furanoate) Compared to Poly(Ethylene Terephthalate). Part 2: Kinetic Sorption. Polymer 2014, 55, 6870-6882. [CrossRef]

5. Knoop, R.J.I.; Vogelzang, W.; van Haveren, J.; van Es, D.S. High Molecular Weight Poly(Ethylene-2,5-Furanoate); Critical Aspects in Synthesis and Mechanical Property Determination. J. Polym. Sci. Part A Polym. Chem. 2013, 51, 4191-4199. [CrossRef]

6. Codou, A.; Moncel, M.; van Berkel, J.G.; Guigo, N.; Sbirrazzuoli, N. Glass Transition Dynamics and Cooperativity Length of Poly(Ethylene 2,5-Furandicarboxylate) Compared to Poly(Ethylene Terephthalate). Phys. Chem. Chem. Phys. 2016, 18, 16647-16658. [CrossRef]

7. Codou, A.; Guigo, N.; van Berkel, J.; de Jong, E.; Sbirrazzuoli, N. Non-Isothermal Crystallization Kinetics of Biobased Poly(Ethylene 2,5-Furandicarboxylate) Synthesized via the Direct Esterification Process. Macromol. Chem. Phys. 2014, 215, 2065-2074. [CrossRef]

8. Guigo, N.; van Berkel, J.; de Jong, E.; Sbirrazzuoli, N. Modelling the Non-Isothermal Crystallization of Polymers: Application to Poly(Ethylene 2,5-Furandicarboxylate). Thermochim. Acta 2017, 650, 66-75. [CrossRef]

9. Guigo, N.; Sbirrazzuoli, N. Thermal Analysis of Biobased Polymers and Composites. In Handbook of Thermal Analysis and Calorimetry; Elsevier: Amsterdam, The Netherlands, 2018; pp. 399-429.

10. Forestier, E.; Combeaud, C.; Guigo, N.; Monge, G.; Haudin, J.M.; Sbirrazzuoli, N.; Billon, N. Strain-Induced Crystallization of Poly(Ethylene 2,5-Furandicarboxylate). Mechanical and Crystallographic Analysis. Polymer 2020, 187. [CrossRef]

11. Forestier, E.; Combeaud, C.; Guigo, N.; Sbirrazzuoli, N.; Billon, N. Understanding of Strain-Induced Crystallization Developments Scenarios for Polyesters: Comparison of Poly(Ethylene Furanoate), PEF, and Poly(Ethylene Terephthalate), PET. Polymer 2020, 203, 122755. [CrossRef]

12. Forestier, E.; Guigo, N.; Combeaud, C.; Billon, N.; Sbirrazzuoli, N. Conformational Change Analysis of Poly(Ethylene 2,5Furandicarboxylate) and Poly(Ethylene Terephthalate) under Uniaxial Stretching. Macromolecules 2020, 53. [CrossRef] 
13. LeBourvellec, G.; Monnerie, L.; Jarry, J.P. Amorphous Orientation and Induced Crystallization in Uniaxially Stretched Poly(Ethylene Terephthalate Glycol). Polymer 1986, 27, 856-860. [CrossRef]

14. Le Bourvellec, G.; Beautemps, J. Stretching of PET Films under Constant Load. II. Structural Analysis. J. Appl. Polym. Sci. 1990, 39, 329-339. [CrossRef]

15. Salem, D.R. Development of Crystalline Order during Hot-Drawing of Poly(Ethylene Terephthalate) film: Influence of Strain Rate. Polymer 1992, 33, 3182-3188. [CrossRef]

16. Ajji, A.; Cole, K.C.; Dumoulin, M.M.; Brisson, J. Amorphous Orientation of Poly(Ethylene Terephthalate) by X-Ray Diffraction in Combination with Fourier Transform Infra-Red Spectroscopy. Polymer 1995, 36, 4023-4030. [CrossRef]

17. Mahendrasingam, A.; Martin, C.; Jaber, A.; Hughes, D.; Fuller, W.; Rule, R.; Oldman, R.J.; MacKerron, D.; Blundell, D.J. TimeResolved X-Ray Wide Angle Scattering Studies of the Effect of Draw Rate and Temperature on the Development of Orientation and Crystallinity in PET. Nucl. Inst. Methods Phys. Res. B 1995, 97, 238-241. [CrossRef]

18. Ajji, A.; Guèvremont, J.; Cole, K.C.; Dumoulin, M.M. Orientation and Structure of Drawn Poly(Ethylene Terephthalate). Polymer 1996, 37, 3707-3714. [CrossRef]

19. Blundell, D.J.; MacKerron, D.H.; Fuller, W.; Mahendrasingam, A.; Martin, C.; Oldman, R.J.; Rule, R.J.; Riekel, C. Characterization of Strain-Induced Crystallization of Poly(Ethylene Terephthalate) at Fast Draw Rates using Synchrotron Radiation. Polymer 1996, 37, 3303-3311. [CrossRef]

20. Middleton, A.C.; Duckett, R.A.; Ward, I.M.; Mahendrasingam, A.; Martin, C. Real-Time FTIR and WAXS Studies of Drawing Behavior of Poly(Ethylene Terephthalate) films. J. Appl. Polym. Sci. 2001, 79, 1825-1837. [CrossRef]

21. Gorlier, E.; Haudin, J.M.; Billon, N. Strain-Induced Crystallisation in Bulk Amorphous PET under Uni-Axial Loading. Polymer 2001, 42, 9541-9549. [CrossRef]

22. Kawakami, D.; Hsiao, B.S.; Ran, S.; Burger, C.; Fu, B.; Sics, I.; Chu, B.; Kikutani, T. Structural Formation of Amorphous Poly(Ethylene Terephthalate) during Uniaxial Deformation above Glass Temperature. Polymer 2004, 45, 905-918. [CrossRef]

23. Kawakami, D.; Hsiao, B.S.; Burger, C.; Ran, S.; Avila-Orta, C.; Sics, I.; Kikutani, T.; Jacob, K.I.; Chu, B. Deformation-Induced Phase Transition and Superstructure Formation in Poly(Ethylene Terephthalate). Macromolecules 2005, 38, 91-103. [CrossRef]

24. Kawakami, D.; Ran, S.; Burger, C.; Avila-Orta, C.; Sics, I.; Chu, B.; Hsiao, B.S.; Kikutani, T. Superstructure Evolution in Poly(Ethylene Terephthalate) during Uniaxial Deformation above Glass Transition Temperature. Macromolecules 2006, 39, 2909-2920. [CrossRef]

25. Kawakami, D.; Ran, S.; Burger, C.; Fu, B.; Sics, I.; Chu, B.; Hsiao, B.S. Mechanism of Structural Formation by Uniaxial Deformation in Amorphous Poly(Ethylene Terephthalate) above the Glass Temperature. Macromolecules 2003, 36, 9275-9280. [CrossRef]

26. Kawakami, D.; Burger, C.; Ran, S.; Avila-Orta, C.; Sics, I.; Chu, B.; Chiao, S.M.; Hsiao, B.S.; Kikutani, T. New Insights Into Lamellar Structure Development and SAXSA/WAXD Sequence Appearance during Uniaxial Stretching of Amorphous Polyethylene Terephthalate above Glass Transition Temperature. Macromolecules 2008, 41, 2859-2867. [CrossRef]

27. Stoclet, G.; Lefebvre, J.M.; Yeniad, B.; Gobius du Sart, G.; de Vos, S. On the Strain-Induced Structural Evolution of Poly(Ethylene2,5-Furanoate) upon Uniaxial Stretching: An in-Situ SAXS-WAXS Study. Polymer 2018, 134, 227-241. [CrossRef]

28. Mao, Y.; Bucknall, D.G.; Kriegel, R.M. Synchrotron X-Ray Scattering Study on Amorphous Poly(Ethylene Furanoate) under Uniaxial Deformation. Polymer 2018, 139, 60-67. [CrossRef]

29. Mao, Y.; Kriegel, R.M.; Bucknall, D.G. The Crystal Structure of Poly(Ethylene Furanoate). Polymer 2016, 102, 308-314. [CrossRef]

30. Menager, C.; Guigo, N.; Martino, L.; Sbirrazzuoli, N.; Visser, H.; Boyer, S.A.E.; Billon, N.; Monge, G.; Combeaud, C. Strain Induced Crystallization in Biobased Poly(Ethylene 2,5-Furandicarboxylate) (PEF); Conditions for Appearance and Microstructure Analysis. Polymer 2018, 158, 364-371. [CrossRef]

31. Chaari, F.; Chaouche, M.; Doucet, J. Crystallization of Poly(Ethylene Terephthalate) under Tensile Strain: Crystalline Development versus Mechanical Behaviour. Polymer 2002, 44, 473-479. [CrossRef]

32. Carr, P.L.; Nicholson, T.M.; Ward, I.M. Mesophase Structures in Poly(Ethylene Terephthalate), Poly(Ethylene Naphthalate) and Poly(Ethylene Naphthalate Bibenzoate). Polym. Adv. Technol. 1997, 8, 592-600. [CrossRef]

33. Mahendrasingam, A.; Blundell, D.J.; Wright, A.K.; Urban, V.; Narayanan, T.; Fuller, W. Observations of Structure Development during Crystallisation of Oriented Poly(Ethylene Terephthalate). Polymer 2003, 44, 5915-5925. [CrossRef]

34. Ran, S.; Wang, Z.; Burger, C.; Chu, B.; Hsiao, B.S. Mesophase as the Precursor for Strain-Induced Crystallization in Amorphous Poly(Ethylene Terephthalate) Film. Macromolecules 2002, 35, 10102-10107. [CrossRef]

35. Welsh, G.E.; Blundell, D.J.; Windle, A.H. A Transient Mesophase on Drawing Polymers based on Polyethylene Terephthalate (PET) and Polyethylene Naphthoate (PEN). J. Mater. Sci. 2000, 35, 5225-5240. [CrossRef]

36. Vigny, M.; Tassin, J.F.; Gibaud, A.; Lorentz, G. Study of the Molecular Structure of PET Films Obtained by an Inverse Stretching Process. Part I: Constant Speed Drawing of Amorphous Films. Polym. Eng. Sci. 1997, 37, 1785-1794. [CrossRef]

37. Asano, T.; Baltá Calleja, F.J.; Flores, A.; Tanigaki, M.; Mina, M.F.; Sawatari, C.; Itagaki, H.; Takahashi, H.; Hatta, I. Crystallization of Oriented Amorphous Poly(Ethylene Terephthalate) as Revealed by X-Ray Diffraction and Microhardness. Polymer 1999, 40, 6475-6484. [CrossRef]

38. Blundell, D.J.; Mahendrasingam, A.; Martin, C.; Fuller, W.; MacKerron, D.H.; Harvie, J.L.; Oldman, R.J.; Riekel, C. Orientation Prior to Crystallisation during Drawing of Poly(Ethylene Terephthalate). Polymer 2000, 41, 7793-7802. [CrossRef]

39. Mahendrasingam, A.; Martin, C.; Fuller, W.; Blundell, D.J.; Oldman, R.J.; MacKerron, D.H.; Harvie, J.L.; Riekel, C. Observation of a Transient Structure Prior to Strain-Induced Crystallization in Poly(Ethylene Terephthalate). Polymer 2000, 41, 1217-1221. [CrossRef] 
40. Quandalle, G. Study and Mechanical Modeling of the Strain-Induced-Crystallization of Polymers: Crosslinked Naturel Rubber and PET. Ph.D. Thesis, PSL Research University, Paris, France, 2017.

41. Federico, C.E.; Bouvard, J.L.; Combeaud, C.; Billon, N. Large Strain/Time Dependent Mechanical Behaviour of PMMAs of Different Chain Architectures. Application of Time-Temperature Superposition Principle. Polymer 2018, 139. [CrossRef]

42. Fabre, V.; Quandalle, G.; Billon, N.; Cantournet, S. Time-Temperature-Water Content Equivalence on Dynamic Mechanical Response of Polyamide 6,6. Polymer 2018, 137. [CrossRef]

43. Billon, N. New Constitutive Modeling for Time-Dependent Mechanical Behavior of Polymers Close to Glass Transition: Fundamentals and Experimental Validation. J. Appl. Polym. Sci. 2012, 125, 4390-4401. [CrossRef]

44. Maurel-Pantel, A.; Baquet, E.; Bikard, J.; Bouvard, J.L.; Billon, N. A Thermo-Mechanical Large Deformation Constitutive Model for Polymers Based on Material Network Description: Application to a Semi-Crystalline Polyamide 66. Int. J. Plast. 2015, 67, 102-126. [CrossRef]

45. Stoclet, G.; Gobius du Sart, G.; Yeniad, B.; de Vos, S.; Lefebvre, J.M. Isothermal Crystallization and Structural Characterization of Poly(Ethylene-2,5-Furanoate). Polymer 2015, 72, 165-176. [CrossRef]

46. Martino, L.; Guigo, N.; van Berkel, J.G.; Kolstad, J.J.; Sbirrazzuoli, N. Nucleation and Self-Nucleation of Bio-Based Poly(Ethylene 2,5-Furandicarboxylate) Probed by Fast Scanning Calorimetry. Macromol. Mater. Eng. 2016, 301, 586-596. [CrossRef]

47. Gorlier, E. Caractérisation Rhéologique et Structurale d'un PET. Application Au Procédé de Bi-Étirage Soufflage de Bouteilles. Ph.D. Thesis, Ecole Nationale Supérieure Des Mines de Paris, Paris, France, 2001.

48. Van Berkel, J.G.; Guigo, N.; Kolstad, J.J.; Sipos, L.; Wang, B.; Dam, M.A.; Sbirrazzuoli, N. Isothermal Crystallization Kinetics of Poly (Ethylene 2,5-Furandicarboxylate). Macromol. Mater. Eng. 2015, 300, 466-474. [CrossRef]

49. Papageorgiou, G.Z.; Tsanaktsis, V.; Bikiaris, D.N. Synthesis of Poly(Ethylene Furandicarboxylate) Polyester Using Monomers Derived from Renewable Resources: Thermal Behavior Comparison with PET and PEN. Phys. Chem. Chem. Phys. 2014, 16, 7946-7958. [CrossRef] [PubMed]

50. Picard, M. Strain Induced Crystallisation During Stretch Blow Moulding of PET. Correlation with Strain Hardening. Ph.D. Thesis, École Nationale Supérieure des Mines, Paris, France, 2008.

51. Williams, M.L.; Landel, R.F.; Ferry, J.D. Temperature Dependence of Relaxation Mechanisms The Temperature Dependence of Relaxation Mechanisms in Amorphous Polymers and Other Glass-Forming Liquids. J. Am. Chem. Soc. 1955, 77, 3701-3707. [CrossRef]

52. Cox, W.P.; Merz, E.H. Correlation of Dynamic and Steady Flow Viscosities. J. Polym. Sci. 1958, 28, 619-622. [CrossRef]

53. Araujo, C.F.; Nolasco, M.M.; Ribeiro-Claro, P.J.A.; Rudić, S.; Silvestre, A.J.D.; Vaz, P.D.; Sousa, A.F. Inside PEF: Chain Conformation and Dynamics in Crystalline and Amorphous Domains. Macromolecules 2018, 51, 3515-3526. [CrossRef] 